\title{
Plateau Tank Apparatus for The Study of Liquid Bridges
}

Andrew Resnick

Cleveland State University, a.resnick@csuohio.edu

J. Iwan D. Alexander

University of Alabama

Follow this and additional works at: https://engagedscholarship.csuohio.edu/sciphysics_facpub

Part of the Physics Commons

How does access to this work benefit you? Let us know!

Publisher's Statement

(C) 1997 American Institute of Physics.

\section{Repository Citation}

Resnick, Andrew and Alexander, J. Iwan D., "Plateau Tank Apparatus for The Study of Liquid Bridges" (1997). Physics Faculty Publications. 249.

https://engagedscholarship.csuohio.edu/sciphysics_facpub/249

This Article is brought to you for free and open access by the Physics Department at EngagedScholarship@CSU. It has been accepted for inclusion in Physics Faculty Publications by an authorized administrator of

EngagedScholarship@CSU. For more information, please contact library.es@csuohio.edu. 


\title{
Plateau tank apparatus for the study of liquid bridges
}

\author{
A. Resnick and J. I. D. Alexander \\ Center for Microgravity and Materials Research, University of Alabama in Huntsville, Huntsville, \\ Alabama 35899
}

(Received 16 September 1996; accepted for publication 22 November 1996)

\begin{abstract}
An apparatus has been constructed and used to study the equilibrium and dynamical behavior of liquid bridges under reduced effective gravity. Liquid bridges are created and manipulated using six independent computer controlled stepper motors which drive linear motion tables. The bridges are visualized with a high magnification coherent Fourier optical system and in an orthogonal view using incoherent white light. By calibrating a density hydrometer and measuring the interfacial energy between the bridge and bath, reliable Bond numbers as low as $10^{-4}$ can be created and held stable for extended periods of time. Dimensional control of the liquid bridges approaches one part in $10^{-4}$ for the aspect ratio and volume. The apparatus has been tested by measuring the static stability limits of axisymmetric bridges and comparing the results with previous theoretical predictions. Experimental error for the apparatus is $\delta \mathrm{Bo} / \mathrm{Bo}=0.03, \delta \Lambda / \Lambda=0.001$ and $\delta V / V$ $=0.001$, where Bo is the Bond number, $\Lambda$ is the aspect ratio of the bridge, and $V$ is the dimensionless (relative) volume of the bridge. (C) 1997 American Institute of Physics.
\end{abstract}

[S0034-6748(97)02403-9]

\section{INTRODUCTION}

A program of study, both theoretical and experimental, has been undertaken to analyze the physics of anchored liquid columns, or liquid bridges. Liquid bridges occupying part of the space between two solid surfaces have been the subject of numerous investigations (see Refs. 1 and 2 for a summary). In particular, research work has focused on the curvature and shape of the liquid profile, the volume of the liquid, the force exerted on the two solids by the bridge, and the stability limits of possible bridge configurations. The goal of constructing this apparatus is to undertake an experimental investigation of the importance of contact line and angle dynamics on the characteristics of dynamic liquid bridges.

There are three dimensionless parameters that determine the physical behavior of the liquid bridge. The dimensionless volume $V$ is defined as $V_{0} / \pi R^{2} L$, where $V_{0}$ is the true volume, $R$ is the radius of the support disk, and $L$ is the height of the bridge; the aspect ratio, defined as $\Lambda=L / 2 R$; and the Bond number $\mathrm{Bo}=\left(\rho_{1}-\rho_{2}\right) g R^{2} / \sigma$, where $\rho_{i}$ is the density of the $i$ th fluid, $g$ is the gravitational constant, and $\sigma$ is the interfacial energy between fluids 1 and 2. A well-known result, the Rayleigh limit, ${ }^{3}$ states that at $\mathrm{Bo}=0$ and $V=1$ the maximum aspect ratio stable with respect to axisymmetric perturbations is $\Lambda=\pi$.

Liquid bridges occur in a variety of physical and biological systems and different aspects of the bridges have been studied by investigators from different fields. Orr et al. ${ }^{4}$ cite a number of applications of the results of liquid bridge research. For example, volume is essential for calculations of water saturation ${ }^{5}$ and connate water estimates in oil and gas reservoirs. ${ }^{6}$ Capillary condensation and evaporation problems in porous media require knowledge of bridge curvatures. ${ }^{7,8}$ Forces exerted by liquid bridges on solids are important in powder metallurgy during liquid phase sintering, ${ }^{9}$ in powder wetting problems, ${ }^{10}$ the deformation of moist porous and unconsolidated granular media, ${ }^{11,12}$ and in adhesion problems. ${ }^{13}$ Liquid bridges and drops are also important factors when considering the positioning of liquid masses using surface tension forces and propellant management in liquid fuel tanks. ${ }^{14}$ More recent applications of liquid bridges include the floating zone method of crystal growth ${ }^{15-17}$ and the biomedical problem of bridge formation in alveoli and the relationship to lung collapse. ${ }^{18,19}$

Experiments are undertaken here using the Plateau or neutral buoyancy method. ${ }^{14}$ This relies on the principle that if two immiscible liquids of equal density are configured such that one envelopes the other then the curvature of the equilibrium interface is a constant. That is, despite the fact that gravity creates a hydrostatic pressure gradient in each liquid, the interface between the two liquids behaves as if gravitational is zero. For nonzero gravity, the density mismatch can be adjusted to cover a range of Bond numbers. In each liquid, the pressure $p_{i}, i=1,2$, satisfies

$$
\left.\operatorname{grad} p_{i}^{*}=0,\right\urcorner
$$

where $p_{i}^{*}=p_{i}+\rho_{i} g z$, and $\rho_{i}$ is the density. At the interface between the two fluids,

$$
p_{1}^{*}-p_{2}^{*}=\left(\rho_{1}-\rho_{2}\right) g z+2 \sigma K .
$$

Here $K$ is the mean curvature of the surface and $\sigma$ is the surface tension. When $\rho_{1}$ and $\rho_{2}$ are equal, the curvature is a constant, and zero-gravity conditions are obtained. Thus, the outer fluid compensates for the hydrostatic pressure gradient along the interface. To obtain a nonzero Bo, the outer bath density can be changed either by adjusting the composition or temperature. ${ }^{20}$

An apparatus has been constructed to perform experiments on liquid bridges in simulated reduced gravity conditions. Several scientific requirements had to be met to ensure accurate and precise results. The primary requirement is to produce accurate and stable Bond numbers over the range $10^{-1}>$ Bo $>10^{-4}$. This range is given by practical density differences between the bridge and bath liquids for the upper 


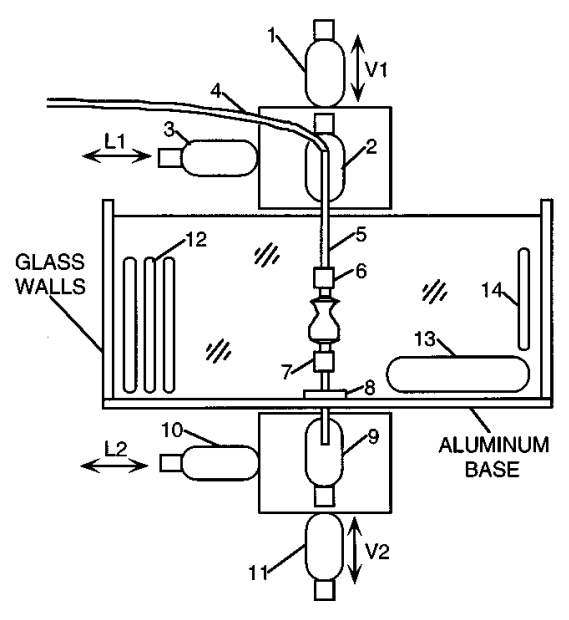

FIG. 1. Plateau tank apparatus: (1) upper vertical displacement motor V1, (2) upper rotational motor, (3) upper lateral displacement motor L1, (4) oil injection line, (5) upper spindle, (6) upper feed disk, (7) lower feed disk, (8) slip-ring gasket, (9) lower rotational motor, (10) lower lateral displacement motor L2, (11) lower vertical displacement motor V2, (12) cooling coils, (13) bath circulator, and (14) heating coil.

bound and measurement precision for the lower bound. This implies control over bath temperature, bath composition, and an accurate method to determine the densities and interfacial energies of the bridge and bath over various temperatures. Lower Bond numbers can also be obtained by using support disks with a smaller radius. Our research involves observing contact line and contact angle behavior during dynamic processes. Consequently, viewing features on the order of tens of microns with light that passes through distorting media is required, along with precise vibrational control of the bridge in several directions, with frequencies ranging from tenths of hertz up to a few hertz, and vibration amplitudes from a fraction of a millimeter to a centimeter. During vibration or rotation, the disk supports must remain parallel, and not exhibit runout or wobble. The support disks must be easily accessible and replaceable, and the tank should be easy to clean. The bridge and bath fluids must be mutually immiscible, and neither should chemically react with the support disks.

Experiments were performed to determine the precision and accuracy of the plateau tank apparatus. One experiment was a calibration of a density hydrometer at various temperatures. In another experiment, the interfacial energy between the bridge and bath solution was measured. An experimental verification of theoretical predictions of static stability limits of axisymmetric liquid bridges were performed. Future experiments will examine the behavior of contact angle motion during axisymmetric and lateral vibrations of liquid bridges.

\section{EXPERIMENTAL SETUP}

\section{A. Apparatus}

The experimental apparatus consists of a neutrally buoyant, or Plateau tank (see Fig. 1) containing $3.8 \times 10^{4} \mathrm{~cm}^{3}$ of bath liquid. The tank is a glass-walled box with a machined aluminum base plate. The base plate has a $5 \mathrm{~cm}$ diam hole for the lower support mechanism and a $0.95 \mathrm{~cm}$ hole for a drainage tube. The apparatus support structure (not shown) consists of $5 \mathrm{~cm}$ diam cast iron pipes filled with sand. The support structure is securely anchored to a $1.22 \times 2.44$ meter optical bench fitted with vibration-damping legs. The lower set of motors are attached to a large aluminum plate that can be adjusted to precisely align the upper and lower support disks. All metal has been painted flat black to reduce reflective interference with the optical systems. The top of the tank is insulated with a Styrofoam slab to reduce evaporative loss of the bath liquid. Bridge supports are threaded onto upper and lower support rods. We have machined a wide variety of sharp-edged disk supports of various materials, sizes, and receding angles. Spherical caps of various materials, wire rings, and disks mounted on cantilever arms have also been fabricated. Each type of support geometry is well suited for a specific physical situation. The support structures are easy to fabricate, and new supports are built as needed for experiments. For most experiments, we use $1 \mathrm{~cm}$ stainless steel sharp-edged disk supports, although the cantilever arm has been used for force measurements and the spherical caps for problems involving a moving contact line.

\section{B. Temperature control}

The experiments were carried out in a temperature controlled laboratory, held at approximately $22{ }^{\circ} \mathrm{C}$. The watermethanol bath was adjusted to the ambient room temperature by using heating or cooling coils. The large thermal mass of the bath, together with the stable ambient room temperature allowed us to hold the temperature of the bath constant to within $0.01{ }^{\circ} \mathrm{C}$ over a $30 \mathrm{~min}$ period, and $0.05{ }^{\circ} \mathrm{C}$ over an $8 \mathrm{~h}$ period. The temperature is measured with a platinum resistance thermometer with a precision of $0.001{ }^{\circ} \mathrm{C}$. Typical experiment times are $2 \mathrm{~h}$ or less. A filtering pump is used to both stir the bath and to remove any foreign material in the solution. All three (pump, heater, and cooling coils) are turned off during experiments. In order to ensure quiescent conditions in the bath during experiments, it is important that a circulator is not used. Schlieren images show that bath temperature nonuniformity is negligible. If the temperature drifts out of the allowed range $\left(\Delta T=0.01{ }^{\circ} \mathrm{C}\right)$ during an experiment, the experiment is stopped and the bath brought to the correct temperature before resuming the experiment. A change in temperature of $\Delta T=0.01{ }^{\circ} \mathrm{C}$ corresponds to a Bond number change of $\Delta \mathrm{Bo}=1.5 \times 10^{-4}$.

\section{Liquid bridge manipulation}

The liquid bridge is entirely created and manipulated using computer control. Our system involves a Pentium series computer running at $133 \mathrm{MHz}$, which communicates with indexers for the motors (Compumotor, series AT 6400). These indexers then communicate with the ac power supply for the motors, which drive linear tables (Parker systems, Daedal division). Our system has 2 AT 6400 cards, one driving four motors and the other driving two motors from a separate indexer. The execution time between consecutive commands is approximately $2 \mathrm{~ms}$ according to the manufacturer. The combination of the motor resolution and the screw thread in the linear tables provides a resolution of 48750 steps $/ \mathrm{cm}$. In addition, there is a joystick interface which can 


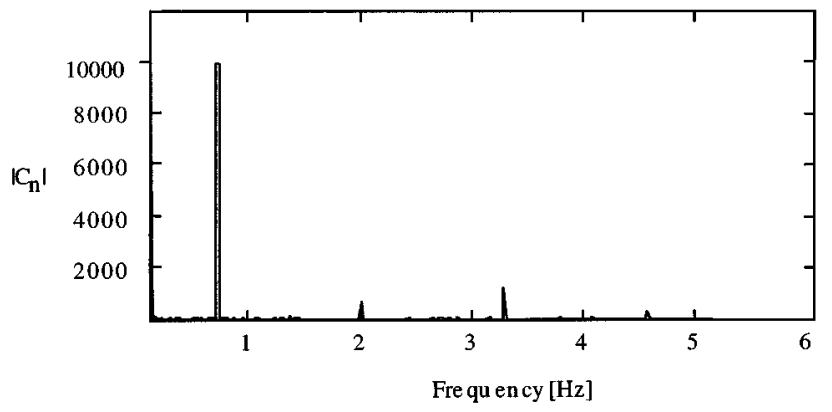

FIG. 2. FFT of encoder motion data. Associated value of Fourier coefficient $\left|C_{n}\right|$ (arbitrary units) vs frequency $(\mathrm{Hz})$

control up to four motors simultaneously. The stepper motors and linear tables were found to have completely repeatable motion, i.e., no measurable backlash. Liquid bridges are created by manual injections of silicone oil with a microsynringe (Hamilton Gastight ${ }^{\circledR}$ series, 50 and $500 \mu \mathrm{l}$ ) while the stepper motors separate the support disks. Typical bridge values range from $10^{-1}$ to $20 \mathrm{~cm}^{3}$, depending on the experiment. The microsyringe injection allows a accurate knowledge of the actual volume, and the high accuracy of the stepper motors provide accurate knowledge of the bridge aspect ratio. Thus, the relative volume of each bridge can be accurately and precisely calculated. Past attempts to automate the syringe failed for various reasons. A glass syringe offers precision, but small angular misalignments between the barrel and plunger caused sticking and the potential for breakage. A plastic syringe could alleviate misalignment, but the rubber-tipped plunger initially deforms instead of travels, resulting in inaccurate volume injection.

Quasisinusoidal motion for a fixed period and acceleration is performed by suitable adjustment of velocity rate as well as distance traveled. A sample Fourier transform of encoder data is shown as an example in Fig. 2. Although there are some higher frequency components, the effect is negligible on our experiments. The particular frequency and amplitude of vibration chosen depend on the particular experiment being performed. Either upper or lower or both support disks may oscillate laterally or vertically, independently or synchronized, depending on the experiment.

\section{Optical setup}

Because the liquid bridge is significantly elevated from the surface of the optical bench, the optics are mounted on elevated optical platforms which are bolted to the optical bench. This increases the stability of the optical components. The optics are mounted onto Data Optics optical rails and 3 -axis carriages. The bridge is viewed in 2 orthogonal directions (Fig. 3). In each case, the bridge is viewed by a CCD camera (Burle ${ }^{\circledR}$, model TC351A) equipped with a $18-105$ $\mathrm{mm}$ zoom lens. In one view, a HeNe laser is used as the light source, and in the other, an incoherent white light source with a diffuser is used as the light source for imaging the entire bridge. The coherent view consists of the laser (15 $\mathrm{mW}$ Spectraphysics Stabilite ${ }^{\mathrm{TM}}$ ) and a $8 \mu \mathrm{m}$ pinhole in a spatial filter for illumination and is used for highmagnification examinations of the contact angle. A lens con-

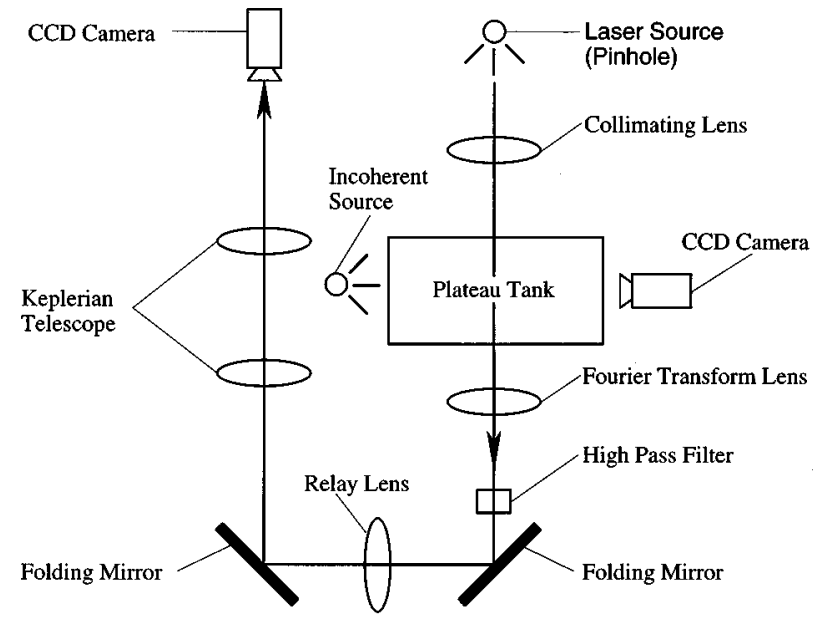

FIG. 3. Optical setup (top view).

verts the spherical wave front to a planar wave front which then passes through the entire Plateau tank. The light is then subjected to high pass frequency filtering ${ }^{21}$ to pass only edges of the bridge silhouette. In addition, magnification is supplied by a second telephoto lens system. It was necessary to clamp the walls of the tank to compensate for the sag induced by the weight of the bath solution, which caused an unacceptable amount of distortion. An image of the Fourier plane without an object is shown in Fig. 4. There is clearly some astigmatism and coma due to residual curvature of the glass walls of the tank. The image from the cameras are digitized by a frame grabber (Coreco TCX). The frame grabber can capture up to $1024 \times 1024 \times 8$ bits for each frame. The images are currently stored and manually processed by a computer program to measure the contact angle. Upgrading

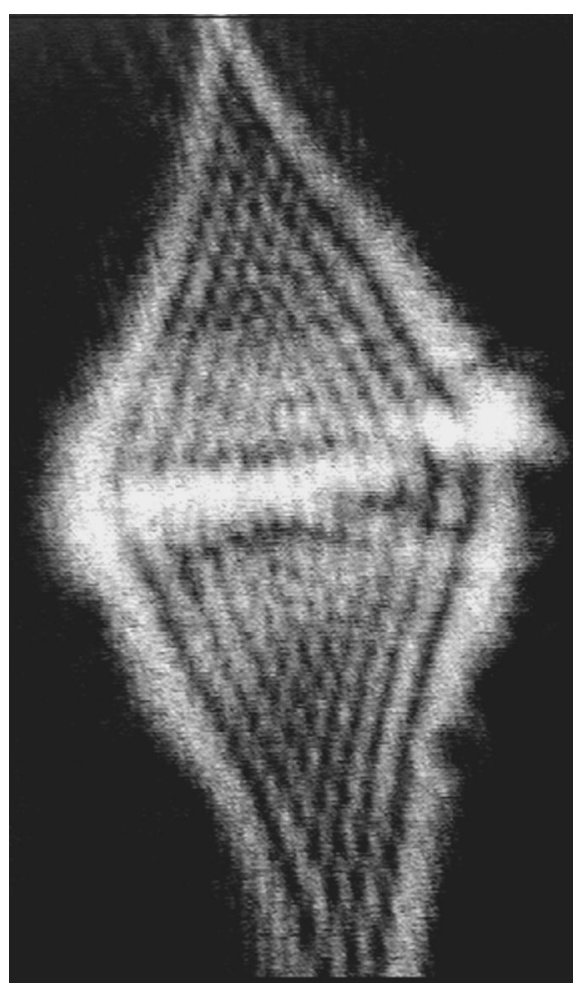

FIG. 4. Focal spot in Fourier plane of optical setup (no bridge present). 

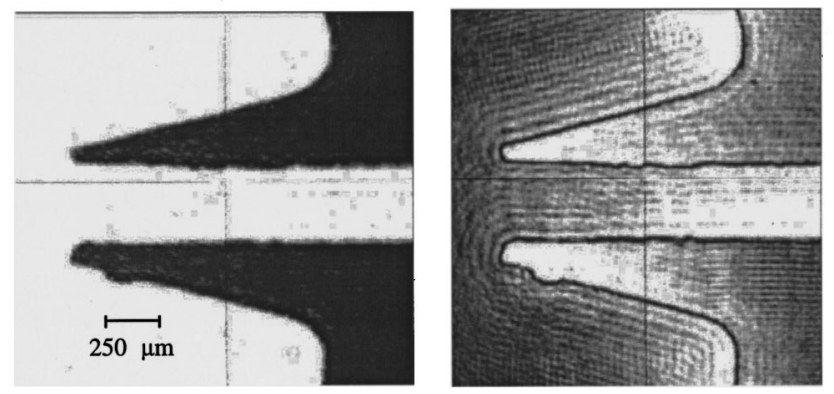

FIG. 5. Sample image showing effect of frequency filtering (no bridge present).

our data acquisition allowed for real-time automatic data acquisition and image processing. The magnifications range from $10 \times$ to $700 \times$ for the coherent view, and $4 \times$ to $11 \times$ for the incoherent view. A typical image of the bridge supports is shown in Fig. 5. Clearly, features on the order of several tens of microns can be resolved, and the aberrations present do not appreciably affect image quality.

\section{E. Materials}

The silicone fluid for the bridge was supplied by Dow Corning. The silicone fluid is a bulk polymerized polydimethylsiloxane with a dynamic viscosity of 5 cs and a measured density of $0.913 \mathrm{~g} / \mathrm{cm}^{3}$ at $22.5^{\circ} \mathrm{C}$. Dow Corning gives the surface tension in air at $25^{\circ} \mathrm{C}$ of $20.9 \mathrm{dyn} / \mathrm{cm}$. The fluid contains not more than $0.5 \%$ volatile materials. The methanol-water bath solution consists of methanol supplied by Fisher Chemical, and was certified to be acetone free, with not more than $0.02 \%$ water content, and has a measured density of $0.786 \mathrm{~g} / \mathrm{cm}^{3}$ at $23{ }^{\circ} \mathrm{C}$. The water was supplied from an in-house Millipore $\mathrm{RO}^{\circledR}$ system coupled with a Milli- $\mathrm{Q}^{\circledR}$ deionizer, producing biological grade type I water with a measured density of $0.996 \mathrm{~g} / \mathrm{cm}^{3}$ at $23{ }^{\circ} \mathrm{C}$. The support disks are machined out of 303-A stainless steel.

\section{PERFORMANCE}

\section{A. Calibration of a density hydrometer at various temperatures}

Accurate and precise knowledge of the bath and bridge density is crucial. Therefore, an experiment was undertaken to calibrate our density hydrometer over a range of temperatures. Silicone oil was used as a density standard, and the condition of neutral buoyancy used to compare measured densities of the bath with independently measured densities of the oil. Our primary concern was the use of the hydrometer over a temperature range $\left(20-30^{\circ} \mathrm{C}\right)$. The hydrometer was an Ertco density hydrometer, 13 in. long, with 0.0005 gradations. The rated accuracy is $2 \%$ at the standard temperature, but we require a substantially greater accuracy over a large temperature range. The hydrometer was placed in a $500 \mathrm{ml}$ graduated cylinder for reading the density. A thermometer was placed in the plateau tank. The temperature was measured using a platinum resistance thermometer, and although the lower limit of resolution of the thermometer is $0.001{ }^{\circ} \mathrm{C}$, local temperature fluctuations forced a practical resolution of $0.01{ }^{\circ} \mathrm{C}$.

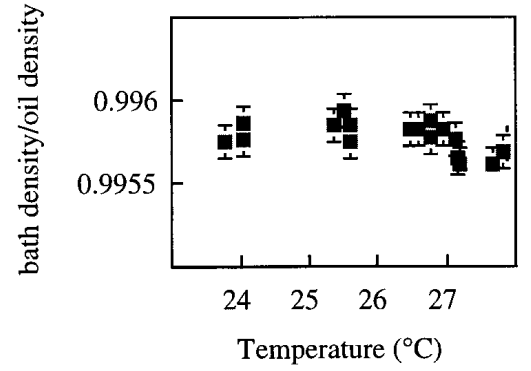

FIG. 6. Temperature dependence of hydrometer.

The temperature dependence of the silicone fluid was measured independently from the hydrometer, using the Archimedes method to weigh an aluminum block submerged in the oil at various temperatures. This method gives the coefficient of expansion for silicone oil as $\Delta \rho / \Delta T=-9.56$ $\times 10^{-4} \mathrm{~g} \mathrm{~cm}^{-3} \mathrm{~K}^{-1}$. This result is less than a $0.1 \%$ difference from the published result by Dow Corning. The size of the hydrometer made use of the Archimedes method impractical and expensive as a special jacketed beaker large enough to hold the hydrometer was not readily available. The plateau tank is kept to a given temperature, within $0.01{ }^{\circ} \mathrm{C}$, and the density of the bath is adjusted by adding either deionized water or methanol until a pulled bridge of aspect ratio 3.12 is stable. The aspect ratio was chosen to be as close to the Rayleigh limit as practical. At this point, the density of the bath is nearly equal to the density of the oil, and a density measurement of the bath is taken and compared against the calculated density of the oil. The bath mixture was mixed continuously, except when pulling a bridge, and was ensured to be homogeneous by use of a Schlieren coherent imaging system. Any inhomogeneities in the bath position create inhomogeneities in the local index of refraction, therefore creating a local lensing effect, which can be easily observed. The graduated cylinder was first filled with bath liquid, then the liquid was expelled as waste to eliminate compositional differences between the fluid in the bath and the fluid in the drain tube, or residual fluid in the cylinder. The graduated cylinder was again filled, and a density measurement taken. The results are shown in Fig. 6. Within the precision of the hydrometer, the variation of temperature does not affect the accuracy of the hydrometer. The data taken by comparing the measured density of the oil to the measured density of the bath is used to increase the accuracy of the hydrometer. Consequently, the density of the bath is known to better than $0.1 \%$.

Because the conditions for neutral buoyancy require a density difference of exactly zero, the time required to achieve exact neutral buoyancy is unacceptably long. In addition, the limit of precision of the hydrometer places a lower limit on how close to neutral buoyancy can actually be measured. Equal numbers of reading were taken with the oil slightly lighter than and slightly heavier than the bath. In this case, it is found that the density difference was equal to the precision of the hydrometer. In order to ensure that the temperature of the measured fluid stayed constant over the period of the experiment, the temperature of the bath was kept close to the ambient temperature of the room. To guarantee that the temperature of the graduated cylinder did not signifi- 


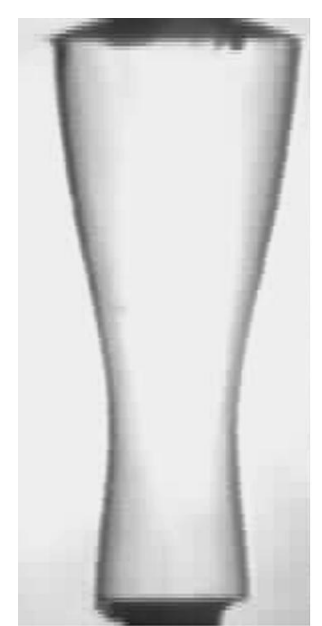

a)

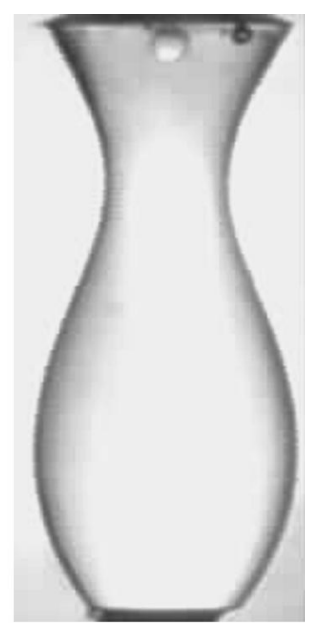

b)
FIG. 7.ᄀ Difference in necking behavior for (a) $\left.\Lambda<\Lambda_{\text {crit }},\right\urcorner$ and (b) $\Lambda>\Lambda_{\text {crit }}$

cantly affect the temperature of the bath portion being measured, when the solution was poured back into the bath, if many Schlieren fringes appeared, the trial was repeated.

\section{B. Measurement of the interfacial energy}

Two methods were used to measure the interfacial energy. One method used theoretical stability limit predictions for liquid bridges between unequal disks, and the second method used the "drop-weight" technique ${ }^{22}$ as a check. It may appear as though theoretical predictions were used to calibrate the experiment which then validated the theory, but this is not the case. The interfacial energy was measured independently using the drop weight method and the values obtained were similar. In addition, the interfacial energy was measured using one theoretical point only, but the experiment covered large portions of the stability curve.

Once the hydrometer was calibrated, the largest uncertainty in the Bond number is in the value of the interfacial energy. Silicone oil has historically been selected for the bridge liquid because the interfacial energy is not sensitive to contamination or temperature. However, because the silicone oil is immersed in a bath of water and methyl alcohol, the

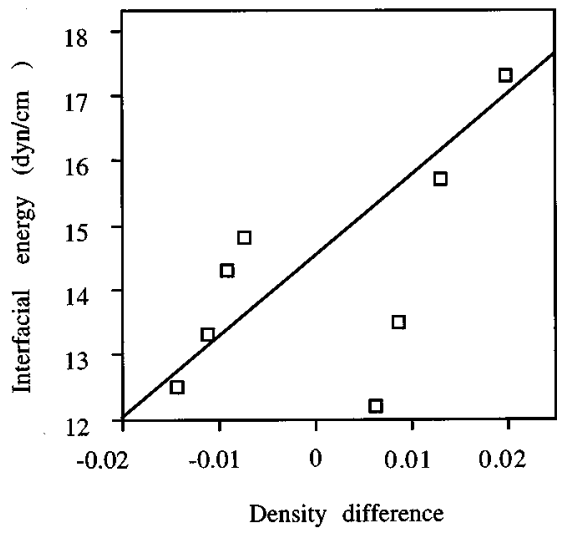

FIG. 8. Interfacial energy vs density difference.

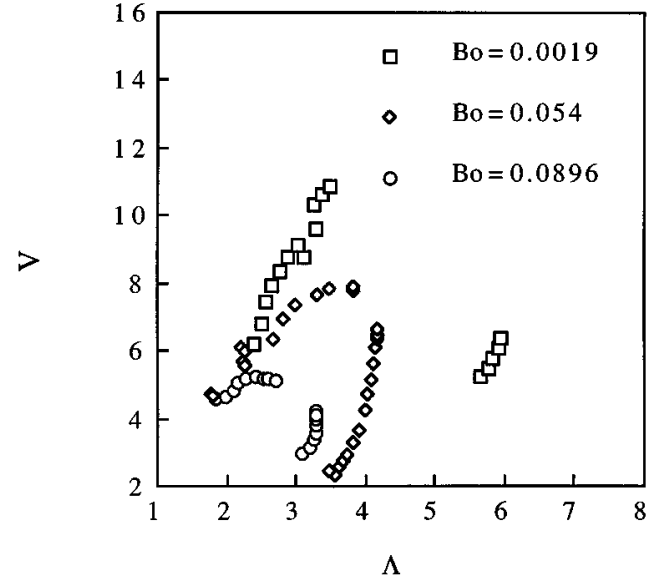

FIG. 9. Stability limits for axisymmetric bridges at various Bond numbers.

value of the interfacial energy will depend on the specific composition of the bath. Consequently, an experiment was performed to measure the interfacial energy of the silicone oil in a bath of varying composition. Theoretical stability limits for unequal disks at $\mathrm{Bo}= \pm 0.100$ have been calculated previously. ${ }^{23}$ The model assumes the contact lines are pinned to the disk edges and remain stationary under perturbations to the liquid bridge. The perturbations are infinitesimal and satisfy the volume preservation condition. In practice, the contact lines are indeed pinned to the disk edges, but the assumption of infinitesimal perturbations may not hold, especially for bridges of large volume and slenderness. However, the use of precision stepper motors and microsyringes to control the volume and slenderness, and the quiescent nature of the bath liquid enable us to meet the perturbation assumption. Along the minimum stability limit for $\mathrm{Bo}>0$, there is a critical aspect ratio $\Lambda_{\text {crit }}$, below which the bridge will neck down near the lower disk, and above which the bridge will neck down near the upper disk (Fig. 7). The value of $\Lambda_{\text {crit }}$ depends crucially upon Bo. The density of the bath was adjusted until the experimental value of $\Lambda_{\text {crit }}$ corresponded with the theoretical value. When the proper aspect ratio was reached, the density was measured with the calibrated hydrometer, and the value of interfacial energy was adjusted until the calculated Bond number was equal to +0.100 . For $\mathrm{Bo}=-0.100$, a slightly different method was used. Because there is no $\Lambda_{\text {crit }}$, an aspect ratio near the maximum stable aspect ratio was used to adjust the density of the bath. Care was taken to be in a region when the value $d V / d \Lambda$ was not large, as small errors in aspect ratio would then cause large errors in minimum stable volume. The data for interfacial energy at $22.0{ }^{\circ} \mathrm{C}$ is shown in Fig. 8. Note the apparent divergence centered around $\Delta \rho=0$. This is an inherent problem with the method, or any method that uses gravity to determine interfacial energy and is not a physical effect. To obtain a value for the interfacial energy, a linear interpolation was taken through the affected region. This gives a value of $\sigma=14.5 \pm 0.5 \mathrm{dyn} / \mathrm{cm}$ at $\mathrm{Bo}=0$, $T=22.0^{\circ} \mathrm{C}$. 


\section{Determination of the stability limits of axisymmetric liquid bridges}

One of the first experiments we performed was a verification of the stability limits of axisymmetric liquid bridges as determined theoretically by Slobozhanin and Perales. ${ }^{24}$ There are two major reasons why this experiment was chosen first. Primarily, verification of such a well-established theoretical study allowed a measure of the accuracy of the apparatus while at the same time optimizing the system. Second, this relatively simple experiment serves as a springboard for more complex experiments, such as a determination of the eigenfrequencies of axisymmetric bridges, as well as studies of nonaxisymmetric bridges. The method for determining the static stability limits involved beginning with a bridge consisting of a well-defined aspect ratio and volume, the slowly deforming the bridge in a known way until the stability limit is reached. This method was done for numerous bridges of different starting configurations, as well as different Bond numbers. In this way, the stability region for each Bond number can be mapped out point by point. In addition, for a particular Bond number, it was desired to determine the transition point between axisymmetric and nonaxisymmetric breaking modes. A Bond number of 0.09 was chosen, which placed the critical aspect ratio $\Lambda \sim 3.4$, and critical volume $V \sim 4.5$.

The results obtained for various Bond numbers are presented in Fig. 9. Each curve represents a portion of the stability limit for a given Bond number. The stability region for each Bond number is enclosed by a set of curve segments which correspond to the stability limits for that Bond number. If a bridge is inside the region bounded by the limit, it is stable with respect to infinitesimal axisymmetric and nonaxisymmetric perturbations. The experiment did not cover the entire stability limit.

The apparatus performed very well, and the lower stability limit was accurately located. The location of the upper stability limit was determined less accurately, because the contact angle does not have complete freedom of motion due to the finite angle of the support disk, ${ }^{25}$ and not due to an inherent error of the measurement. The axisymmetric to nonaxisymmetric transition was located at the theoretically predicted point.

\section{SYSTEM ERROR}

The uncertainty of the Bond number is the most important to know. The error is calculated with two independent methods. The first method uses the uncertainty in density, disk radius, interfacial energy, and " $g$ " to calculate the uncertainty in Bond number.

$$
\left.\frac{\delta \mathrm{Bo}}{\mathrm{Bo}}=\left[\left(\frac{\delta \Delta \rho}{\Delta \rho}\right)^{2}+\left(\frac{\delta g}{g}\right)^{2}+\left(\frac{2 \delta r}{r}\right)^{2}+\left(\frac{\delta \sigma}{\sigma}\right)^{2}\right]^{1 / 2} \cdot\right\urcorner
$$

This gives $\delta \mathrm{Bo} / \mathrm{Bo}=3.45 \times 10^{-2}$. The largest error is in interfacial energy, followed by error in disk radius, then den- sity, and uncertainty in $g$ is last. The second method uses the relation between maximum aspect ratio of a right circular cylindrical bridge as a function of Bond number to calculate the error in Bo.

$$
\left.\Lambda_{\max }=\pi\left[1-\frac{3^{4 / 3}}{2} \mathrm{Bo}^{2 / 3}\right] \cdot\right\urcorner
$$

The linear actuators allowed precise determinations of the linear dimensions of the system. However, the support disks can not start out at zero height, but instead are placed very close together, a few microns apart, to allow the oil room to flow out. The typical initial separation is $0.2 \mathrm{~mm}$, as measured $\urcorner$ with $\urcorner$ high $\urcorner$ magnification. $\urcorner$ For $\urcorner \Lambda_{\max }=3.12$, $\mathrm{Bo}=2.4 \times 10^{-4}$, and $\delta \mathrm{Bo} / \mathrm{Bo}=5.36 \times 10^{-6}$. Therefore, very low Bond number conditions can be created, but the accuracy of measurement of $\mathrm{Bo}$ is $\delta \mathrm{Bo} / \mathrm{Bo}=3.45 \times 10^{-2}$.

\section{ACKNOWLEDGMENTS}

Mr. Resnick gratefully acknowledges NASA for providing a GSRP Grant, Contract No. NGT-51355, under which this work was performed. The authors also gratefully acknowledge Dr. Alexandre Fedoseyev and Dr. William Kaukler for helpful discussions and assistance. We also thank Dow Corning for providing the silicone oil.

${ }^{1}$ V. P. Mehrolta and K. V. Sastry, Powder Technol. 25, 203 (1980).

${ }^{2}$ J. M. Perales and J. Meseguer, Phys. Fluids A 4, 1110 (1993).

${ }^{3}$ L. Rayleigh, Proc. London Math. Soc. 10, 71 (1879).

${ }^{4}$ F. M. Orr, L. E. Scriven, and A. P. Rivas, J. Fluid Mech. 67, 723 (1975).

${ }^{5}$ W. B. Haines, J. Agric. Sci. 15, 529 (1925).

${ }^{6}$ N. R. Morrow, J. Can. Pet. Tech. 10, 38 (1971).

${ }^{7}$ R. Defay and I. Prigogine, in Surface Tension and Absorption (Wiley, New York, 1966), pp. 217-227.

${ }^{8}$ D. H. Everett, in The Solid-Gas Interface, edited by E. A. Flood (Dekker, New York, 1967), Vol. 2, pp. 1105-1113.

${ }^{9}$ R. B. Heady and J. W. Cahn, Metall. Trans. 1, 185 (1970).

${ }^{10}$ P. C. Carmen, J. Phys. C 57, 56 (1953).

${ }^{11}$ W. B. Haines, J. Agric. Sci. 17, 264 (1927).

${ }^{12}$ R. A. Fisher, J. Agric. Sci. 16, 492 (1926).

${ }^{13}$ A. D. Zimon, Adhesion of Dust and Powder (Plenum, New York, 1969).

${ }^{14}$ I. M. Martinez, J. M. Haynes, and D. Langbein, in Fluid Sciences and Material Sciences in Space (Springer, Berlin, 1987), pp. 53-80, see also D. Langbein, J. Phys. Condens. Matter 2, SA491-SA498 (1990).

${ }^{15}$ S. R. Coriell, S. C. Hardy, and M. R. Cordes, J. Colloid Interface Sci. 60, 126 (1977).

${ }^{16}$ C. H. Chun, Acta Astron. 7, 479 (1980).

${ }^{17}$ D. Langbein, J. Cryst. Growth 104, 47 (1990).

${ }^{18}$ R. B. George et al., in Chest Medicine, Essentials of Pulmonary and Critical Care Medicine (Williams and Wilkins, Baltimore, 1990).

${ }^{19}$ M. Johnson et al., J. Fluid Mech. 233, 141 (1991).

${ }^{20}$ B. J. Lowry and P. H. Steen, AIAA preprint (unpublished).

${ }^{21}$ J. Goodman, Introduction to Fourier Optics (McGraw-Hill, New York, 1988).

${ }^{22}$ A. W. Adamson, Physical Chemistry of Surfaces, 5th ed. (Wiley, New York, 1990).

${ }^{23}$ L. A. Slobozhanin, J. I. D. Alexander, and A. Resnick, in Proceedings of the 2nd Symposium on Fluids in Space, Naples, Italy, April 1996 (unpublished).

${ }^{24}$ L. A. Slobozhanin and J. M. Perales, Phys. Fluids 5, 1305 (1993).

${ }^{25}$ D. C. Dyson, Phys. Fluids 31, 229 (1988). 
Review of Scientific Instruments is copyrighted by AIP Publishing LLC (AIP). Reuse of AIP content is subject to the terms at: http://scitation.aip.org/termsconditions. For more information, see http://publishing.aip.org/authors/rights-and-permissions. 